\title{
Entrelacs
}

Cinéma et audiovisuel

18 | 2021

Laurent Roth, cinéaste

\section{Laurent Roth, un cinéma de l'éblouissement}

Dario Marchiori

\section{OpenEdition}

Journals

Édition électronique

URL : https://journals.openedition.org/entrelacs/6057

DOI : $10.4000 /$ entrelacs.6057

ISSN : 2261-5482

Éditeur

Éditions Téraèdre

Référence électronique

Dario Marchiori, «Laurent Roth, un cinéma de l'éblouissement », Entrelacs [En ligne], 18| 2021, mis en ligne le 13 décembre 2021, consulté le 15 décembre 2021. URL : http://journals.openedition.org/ entrelacs/6057 ; DOI : https://doi.org/10.4000/entrelacs.6057

Ce document a été généré automatiquement le 15 décembre 2021.

Tous droits réservés 


\title{
Laurent Roth, un cinéma de l'éblouissement
}

\author{
Dario Marchiori
}
«Kol-no'a : une voix qui bouge et qui appelle à naître. N'est-ce pas avec cette nomination paradoxale du cinéma que nous vient cette profonde certitude : qu'il y a pour passer par les images, une autre voie ${ }^{1}$ ?» Laurent Roth

1 Il y a, au cinéma, des cinéastes qui explorent les limites du cinéma, pour mieux en dévoiler les puissances. Laurent Roth est de ceux-là, bricoleur d'un dialogue risqué entre la parole et l'image, entre le sujet et le monde. Cet entre-deux incertain, Roth le cherche et le retrouve à chaque film, tel le «point d'intersection » dont parle Bresson dans une note sur le cinématographe : «Les grandes batailles, disait le général M..., se livrent presque toujours aux points d'intersection des cartes d'état-major ${ }^{2}$ ». Roth luimême relaie cette citation dans un tout autre contexte, mais elle me paraît exprimer quelque chose de plus fondamental sur son positionnement de cinéaste, ou plus largement d'auteur. À partir notamment de ses premiers films, je m'intéresserai à cet entre-deux créatif, que je poserai comme l'espace d'une mise en tension, d'un bouleversement et d'un éblouissement, qui rejoint par d'autres chemins l'idée du sublime (notamment lyotardien) développée par Roger-Philippe Bertozzi ${ }^{3}$ : dépassée, l'imagination s'éblouit face au spectacle de la nature, ou bien de l'art.

\section{L'éblouissement}

2 Heureusement pour ses spectateurs, Laurent Roth est un cinéaste atypique, dont l'œuvre cinématographique n'est d'ailleurs qu'un volet parmi de multiples activités. Au cinéma, il explore surtout et notamment la forme brève. De courts poèmes, tels Ave Maria (1987) ou Écoute Israël (2014), mais également des films processuels, qui sont à la fois le déclencheur et la trace d'un geste auto-analytique, comme J'ai quitté l'Aquitaine 
(2005), ou encore Ranger les photos (1998/2009). Cette brevitas penche du côté de l'intensité esthétique propre au cinéma expérimental ${ }^{4}$, et explore tout particulièrement la relation entre l'image et la parole. Aussi, l'expérimentation du réel est au cœur du processus de création de Roth, à la croisée de la fiction et du documentaire, mais également à la recherche de manières de raconter et de formes filmiques singulières.

3 L'éblouissement - cet aveuglement lié à un éclat de lumière, cette stupeur qui crée un malaise et «affaiblit» le sujet, d'après l'étymologie ${ }^{5}$ - Laurent Roth le figure littéralement, à la fin de deux de ses premiers films, Marie ou le retour (1984) et Les Yeux brûlés (1986). Dans l'un comme dans l'autre, cet instant de dépossession se rattache à l'expérience-limite de la guerre: dans le premier, c'est le retour du père officier de marine, accompagné d'un bruit assourdissant d'hélicoptère, qui provoque l'éblouissement de l'enfant, d'autant plus puissant qu'il se traduit par un faux raccord (fig. 1 et 2 ); dans le deuxième, le fondu au blanc dissout le très gros plan de la comédienne, Mireille Perrier, qui rentre de Roissy en voiture, en larmes, pâle et annihilée, après les dernières images de guerre à la fois horribles et majestueuses (fig. 3).

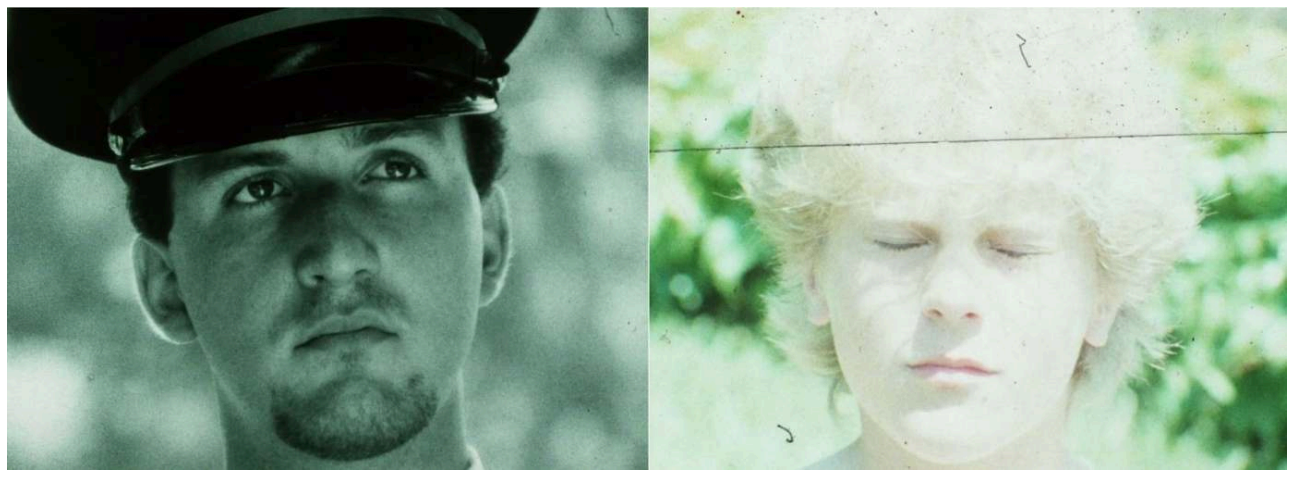

Fig. 1 et 2 


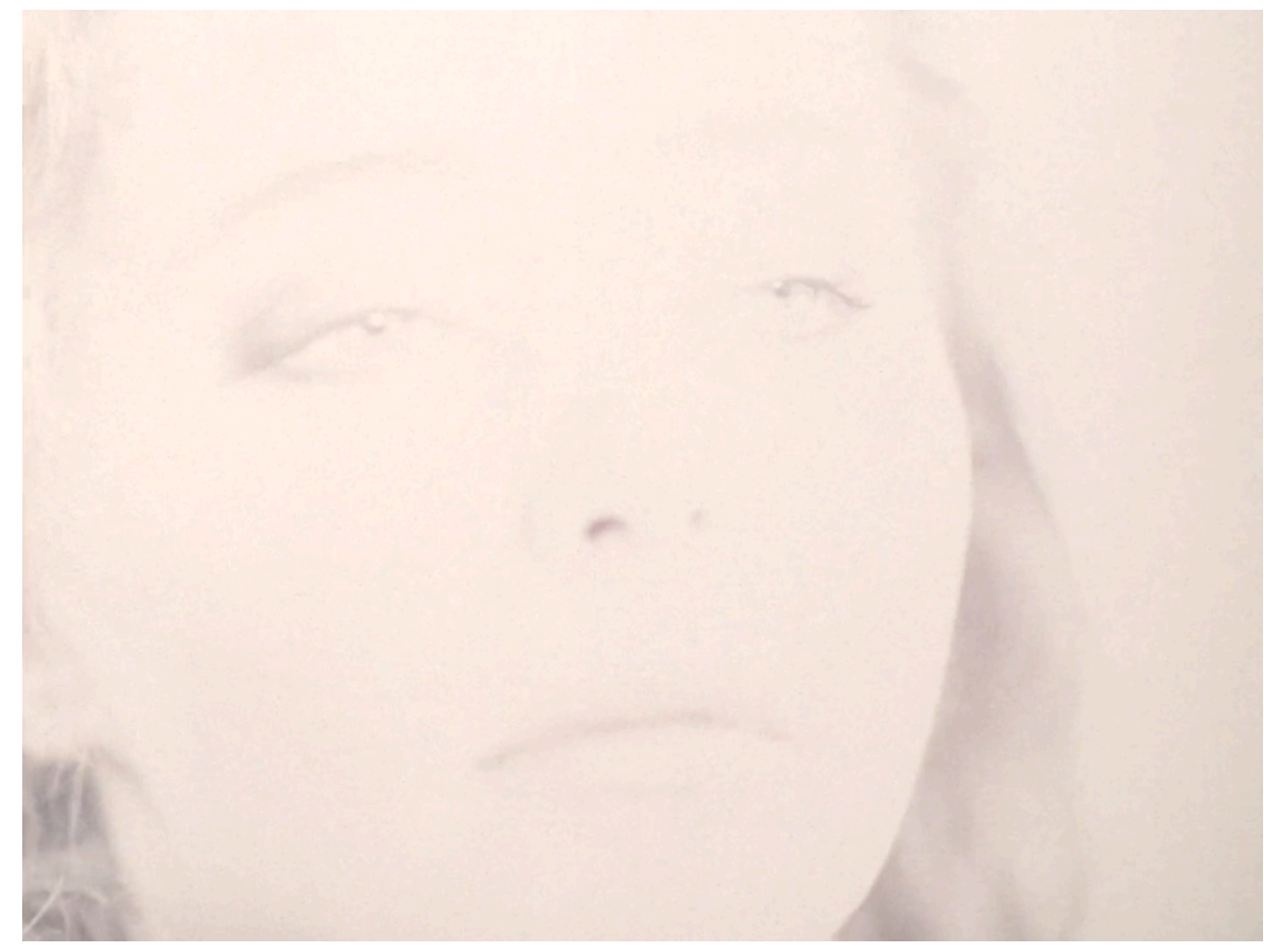

Fig. 3

4 Selon une tradition bien présente dans le monde occidental, il y a un lien fort entre le savoir (ou le savoir-faire) et la cécité : les mythes grecs nous relatent des figures plus ou moins célèbres, de Tirésias à đEdipe, de Phinée à Démodocos, et des cas semblables se retrouvent également dans d'autres cultures, y compris sans lien avec la cécité comme dans l'histoire biblique de la chute. L'éblouissement peut être sans retour, tandis que les deux films de Roth laissent ouverte la fin du récit, tel un éblouissement narratif qui laisse le spectateur sans mots, sans suite, sans conclusion. Dès lors, la fin ouverte éblouit et interpelle.

5 L'éblouissement, dans Les Yeux brûlés, advient également au moyen de la ponctuation qui scande le film, des titres de « chapitres » annonçant les différents sujets abordés, du départ à l'agonie, en passant par l'amitié. L'apparition des cartons marque tout le contraire d'une pause : un écran blanc interrompt le flux d'images et devient la surface d'un autre surgissement, celui des images vidéo retravaillées par l'arrêt, le ralentissement, la déformation (fig. 4 et 5). L'informe agresse et démange la figuration, renversant la beauté des images tournées en $35 \mathrm{~mm}$ dans l'aéroport de Roissy, propres, nettes, aseptiques et par là même inquiétantes (fig. 6), hétérogènes en tout point par rapport à la "saleté » des images de guerre. Ces images vidéo figurent le lieu d'une émergence matérielle, violente, puissante, mais suggèrent également une déperdition abyssale, propre à l'image de guerre, qui se situe à la fois au cœur de l'horreur et toujours en-deçà de l'intournable. Un geste en trop, un coup du hasard exposeraient l'opérateur à la mort, la caméra à sa défaite: «il n'est d'image que faite en "coproduction" avec la $\operatorname{mort}^{6}{ }^{\star}$. L'éblouissement, c'est l'origine de la vision, le surgissement de l'image mais aussi son horizon, sa catastrophe, son point-limite avant l'absence. 


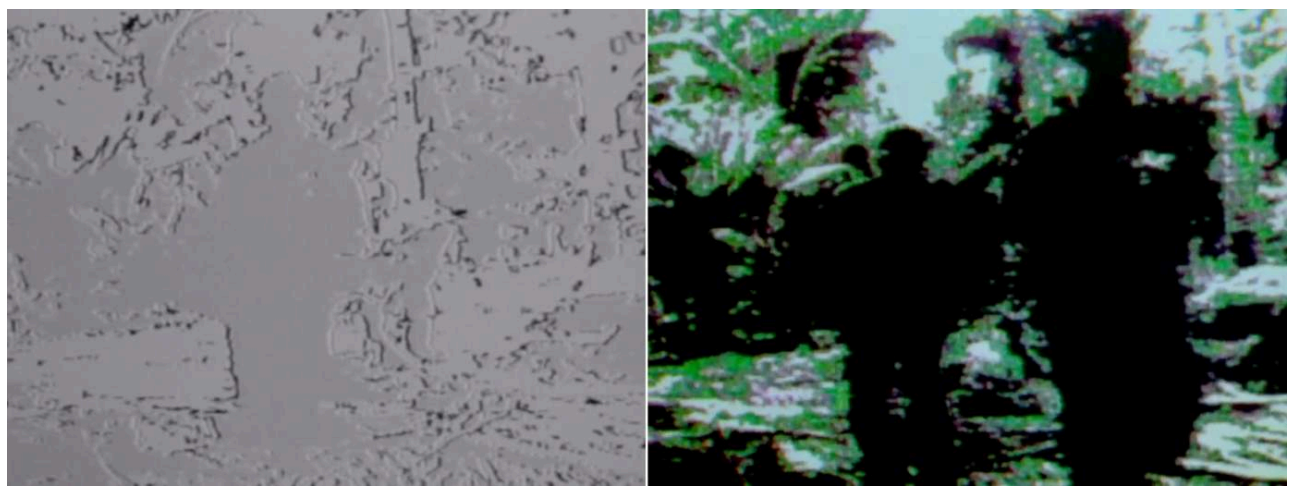

Fig 4,5

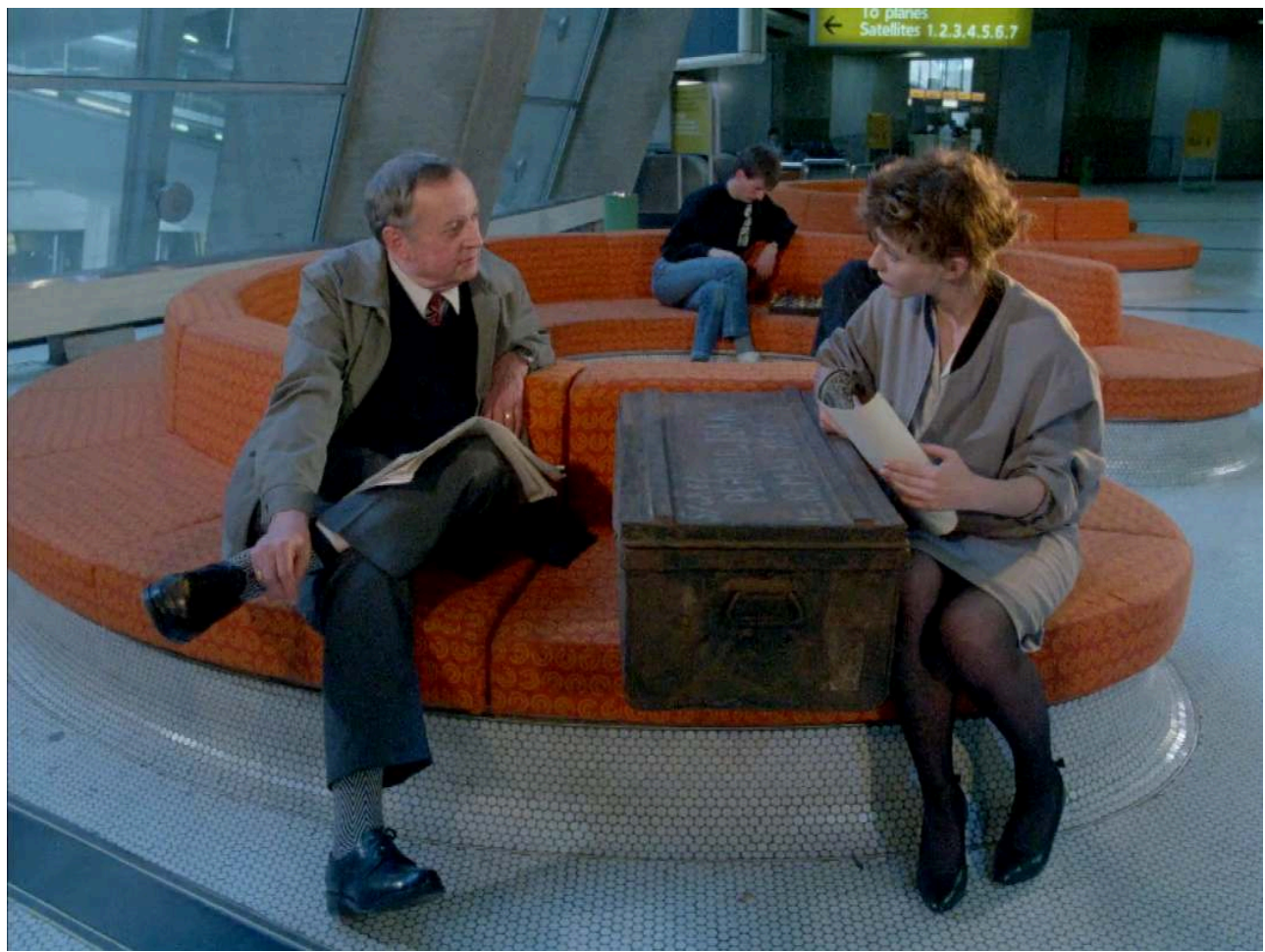

Fig 6

6 «L'éblouissement renvoie le monde au chaos, à son indétermination et à son invisibilité premières $^{7}$ » : en écrivant sur la photographie, Régis Durand prolonge ici une théorie de l'image qui renvoie à Platon, dont il cite le célèbre passage de La République, VII : « Si on le forçait à regarder la lumière même, ne crois-tu pas que les yeux lui feraient mal et qu'il se déroberait aux choses qu'il peut regarder ${ }^{8}$ ? ». L'éblouissement défie l'image en exacerbant à la fois ses limites et ses puissances, y compris destructrices. Qu'est-ce que l'image de la guerre sinon la contradiction brûlante et toujours ouverte entre l'image manquante et l'image qui brûle les yeux, entre l'image aveugle et l'image aveuglante? Laurent Roth semble faire état de cette tension dans un entretien ${ }^{9}$ qu'il donne à l'époque des Yeux brûlés, en 1986: d'une part, il souligne la "fascination pour ce qui met la quête en échec", à savoir que "l'invisibilité même de ce qui est regardé (l'ennemi, le feu) semble être l'article de foi du reporter de guerre. Il se nourrit de l'invisibilité comme de son Dieu »; d'autre part, il s'inscrit dans la tradition biblique de l'interdiction de l'image, en nous offrant l'une des visions les plus ambitieuses et puissantes de l'art cinématographique : «Le commandement est la question ultime de 
l'œuvre d'art, parce qu'il met la parole à l'origine de la vision, le "Tu ne feras pas d'image" à la source de la représentation. Cela seul fait vivre le cinéma ». Quoi qu'on en pense en termes théologiques, en travaillant l'écartèlement entre transcendance et immanentisme, Roth pose un défi radical au cœur de son film - voire de tous ses films : qu'est-ce que peut une image? Et de manière complémentaire, qu'est-ce que peut la parole?

7 Les puissances de l'image sont écrasantes aussi bien pour l'iconolâtre que pour l'iconoclaste ${ }^{10}$. À son insu, l'opérateur de guerre serait l'idolâtre d'un deus absconditus, condamné à produire des images de l'absence, que seule la parole peut - sans doute racheter : tout le projet des Yeux brûlés est contenu dans cette tentative de donner la parole, de parachever l'expérience-limite par l'expression de cette limite même. Seule la parole peut traduire l'infilmable en indicible, remettre la parole au cœur de l'œuvre, exprimer qu'il y a de l'inexprimable, lui donner une forme qui puisse supporter les limites constitutives de la représentation. S'il explore l'insuffisance des images à rendre compte de l'horreur, il le fait non pas par une démission de l'image, mais en reconnaissant et en mettant à l'épreuve son pouvoir comme son impuissance. Dans Les Yeux brûles, Roth opère un double mouvement : il construit un dispositif parafictionnel pour réfléchir sur les images de guerre, pour les explorer, mais il leur reconnaît et il en restitue un pouvoir de fascination troublant. Ce double mouvement d'attrait et de mise à distance n'est qu'apparemment paradoxal, car il s'appuie sur une seule et même considération de la puissance des images.

8 Jusqu'ici, nous avons posé les éléments d'une dialectique de l'image, et de notre rapport à celle-ci. Cependant, le dispositif des Yeux brûlés va plus loin, sur un autre niveau. Les images d'archives, tant photographiques que cinématographiques, sont là pour ellesmêmes, mais thématisent aussi la question du point de vue du photographe ou de l'opérateur. Se définit alors un deuxième niveau d'enchâssement, constitué par les entretiens où les opérateurs réfléchissent à leur pratique par le filtre de la mémoire. Roth ajoute au dispositif un troisième niveau d'enchâssement en intégrant une interlocutrice, l'actrice Mireille Perrier, qui a un rôle opératoire (interviewer les opérateurs) mais incarne aussi la première spectatrice de ces images et des récits qui les accompagnent. Son corps à l'écoute est bien coprésent au récit, réagissant tout à la fois en tant qu'actrice et en tant que personnage. On remarquera que ce double rôle de médiatrice et de réceptacle d'images et de mots se retrouve dans bon nombre de films du cinéaste, sous les traits d'un autobiographisme détourné par la fiction, ou bien par le truchement de la lecture (fig. 7), comme dans Écoute Israël et La Joie (2015). 


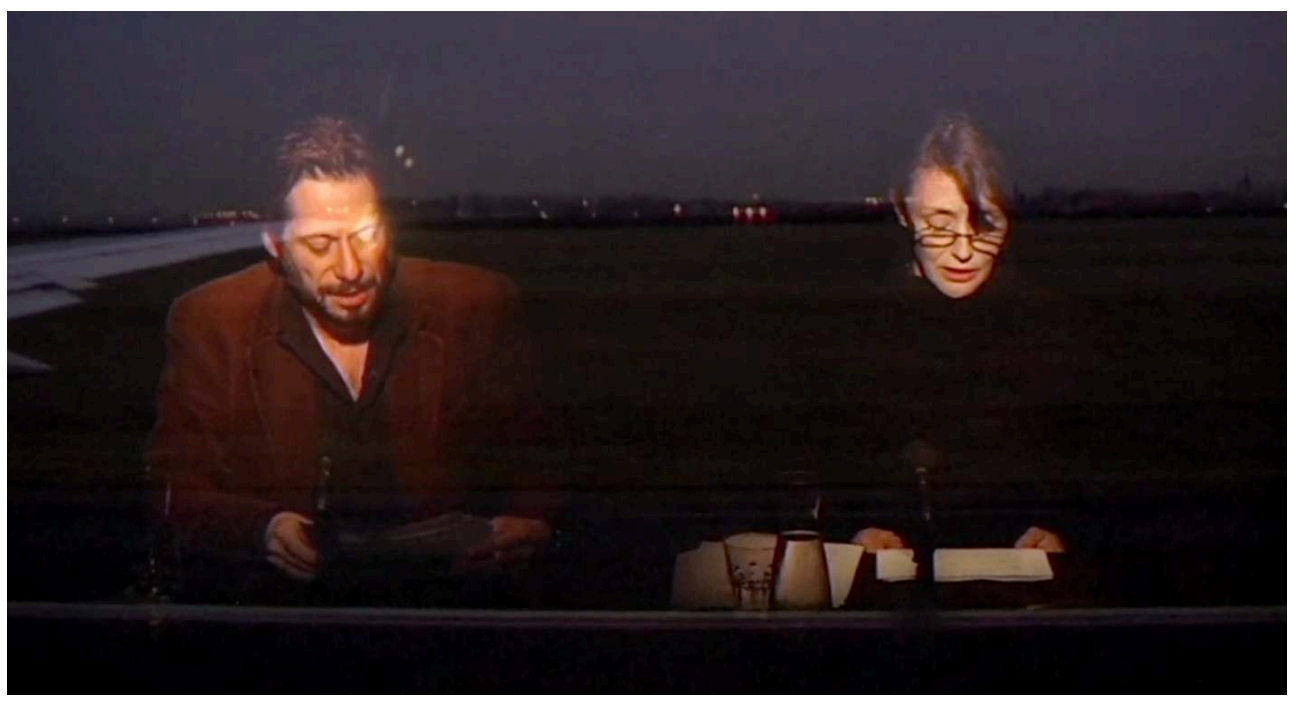

Fig. 7

Dans Les Yeux brûlés, un dernier personnage vient s'ajouter à la série d'enchâssements, bien moins commenté que les trois autres, à savoir le photographe. Pure fonction métadiscursive, il thématise le regard des photographes de guerre, tout en l'inversant car son objet du regard et peut-être du désir est tout à fait visible, mais pas moins mystérieux et finalement inatteignable, le corps de l'actrice. Plus profondément, le photographe représente aussi un double de la mise en scène du film, renvoyant au geste cinématographique de Laurent Roth, pour mieux le cacher tout en l'exposant. Ce parallélisme est souligné à la fin du film, quand le regard de Mireille Perrier se dirige vers le haut où nous avons l'habitude de situer le contre-champ du photographe, alors que celui-ci est assis derrière elle, si bien que le regard s'adresse directement à la caméra en interpellant tout à la fois la mise en scène et le spectateur (fig. 8). Exemple parmi bien d'autres de l'emploi de discontinuité et faux-raccord, ce montage participe à construire une poétique de l'écart et de l'entrechoc ponctuels - et d'autant plus puissants. La béance du tissu narratif et métanarratif (le photographe comme alter ego du réalisateur) soumet le partage entre fiction et documentaire à une réflexivité qui vise plus généralement l'image photo-cinématographique. 


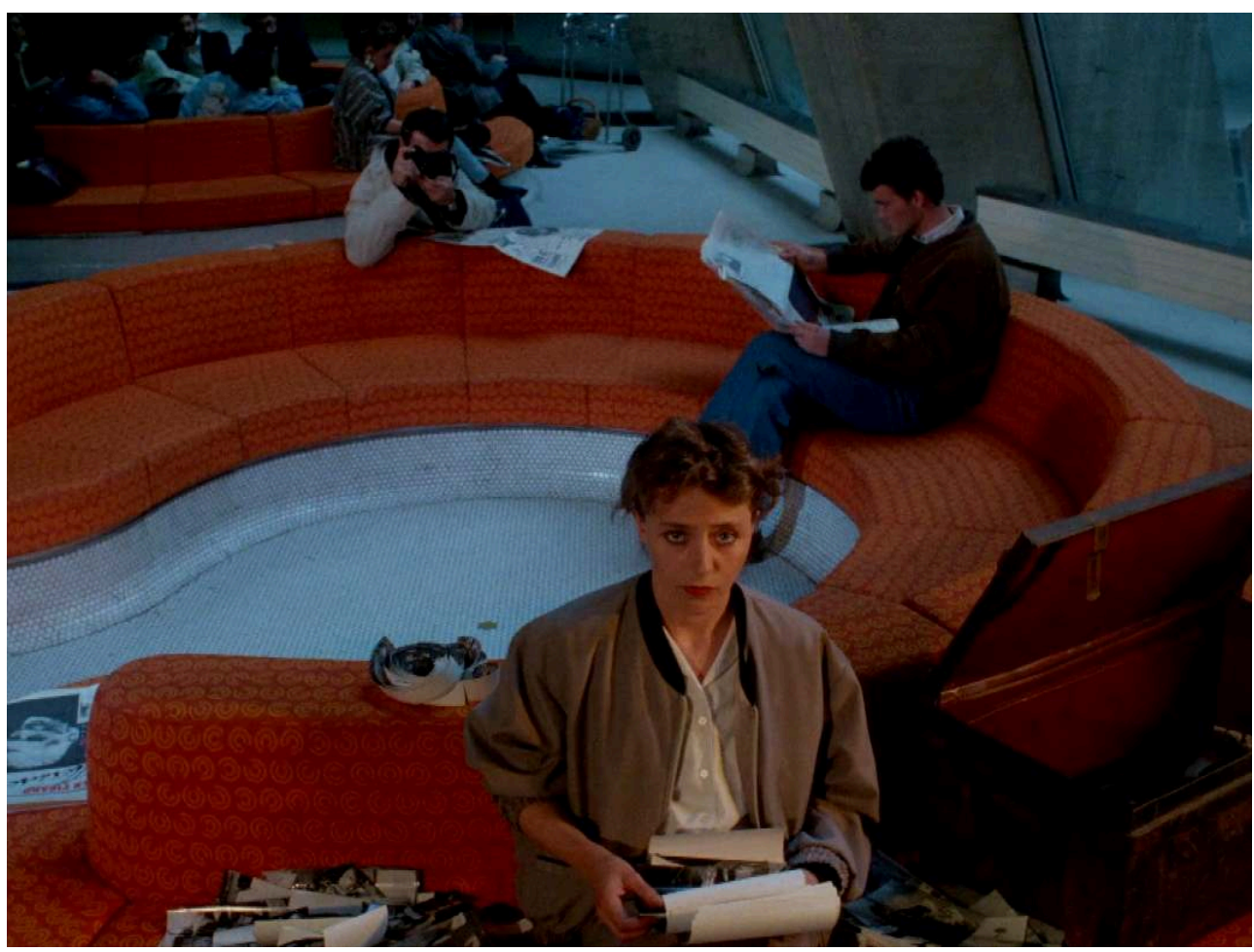

Fig. 8

10 Au cœur du dispositif stratifié que je viens de décrire, Mireille Perrier représente aussi l'alter ego du cinéaste, qui travaille avec lui et pas seulement pour lui, dans une forme d'intersubjectivité que Roth semble chercher dans tous ses films, en laissant une place centrale au corps de l'acteur, du lecteur, de l'interlocuteur - des termes qui se déclinent ici au féminin, en instaurant un dialogue entre les sexes dont on retrouve la trace dans un bel entretien avec Dominique Cabrera ${ }^{11}$. Alter ego dialogique donc, Mireille Perrier est située dans la même position inconfortable que le cinéaste et constitue une figure bien plus complexe et moins cérébrale par rapport au photographe qui lui rôde autour. Elle évolue dans l'entre-deux du passeur, mais elle devient également une enquêtrice en territoire masculin, et volontiers machiste. Cette position fondamentalement mouvante et instable correspond très exactement à celle que cherche Laurent Roth dans et par ses films.

11 Arrêtons-nous un instant sur Modèle depuis toujours (1988), court-métrage d'une dizaine de minutes tourné en vidéo. Inspiré par les 24 portraits d'Alain Cavalier (1987/1991), le film de Roth aborde un métier très particulier, celui du modèle. Si le film a comme condition d'existence une relation amoureuse entre le cinéaste et Claire-Marie Magen, on y retrouve aussi la question du médiateur. Le corps sans parole représente pour les élèves un écran de projection, qui ne laisse rien transparaître de son activité imaginative pendant la pose mais qui n'a de cesse de se promener dans ses pensées comme le raconte la voix off de la modèle. Il y a de l'infigurable, une fois de plus, et au cœur du modèle niche une opacité absolue qui enrichit le créateur (ici le peintre) et qui établit un espace de liberté infini, car indéfini et indéfinissable, pour le modèle. Nous ne sommes pas loin du rôle joué par Mireille Perrier dans Les Yeux brûlés, alors qu'une telle réflexion sur le modèle ne peut que s'inscrire dans la filiation bressonienne. Sans incarnation, pas d'image : Modèle depuis toujours nous permettra désormais de prolonger et creuser l'imbrication entre le corps et l'image dans le cinéma de Laurent Roth. 


\section{L'acte d'image} propre de l'image qui dépasse le simple artefact et semble lui conférer une vie propre, une capacité d'action. D'après l'historien de l'art, qui s'appuie sur la notion d'« acte de langage " théorisée par John Austin ${ }^{13}$, il existerait trois types d'actes d'images: schématique, substitutif et intrinsèque. Le premier se définit par l'introduction du charnel dans l'image (on peut songer à Modèle depuis toujours); l'acte d'image substitutif opère un échange entre l'image et le corps, par exemple dans le domaine du sacré (Ave Maria exprime cela non pas par le corps de Marie, mais par les choses du monde que le film met en avant, de la nature aux objets); le troisième, l'acte d'image intrinsèque, serait lié aux puissances propres de la forme, à la matière constitutive de l'image (tels les surgissements d'images par la surimpression, dans La Joie - voir plus haut fig. 7). image, dont la fixité ne fait qu'accroitre paradoxalement la proximité entre l'image fixe et le corps figé par la pose, entre la vie et son image ("J'avais la vie à donner », dit-elle). Être modèle, cela signifie incarner une potentialité d'images. De plus, la jeune femme est modèle « depuis toujours », comme si la temporalité s'enroulait sur elle-même pour transcender une deuxième fois toute image produite, à la faveur d'un être qui reste à l'état virtuel, par-delà ses actualisations multiples, par-delà la vie du sujet qu'elle incarne : «C'est la vie que je porte qui est à saisir. Elle vient de bien avant moi, et elle continuera bien après moi. Je suis un maillon, ou un reflet, rien d'autre ». Au même moment, par un contraste saisissant, la caméra suit le corps féminin avec un soin et une sensualité extrêmes, en singularisant la beauté d'un corps alors même qu'il se dérobe par l'humilité du propos. Cette déliaison entre l'image et les pensées verbalisées de la modèle renvoie, une fois de plus, à la limite constitutive de toute image. D'autre part, la voix sollicite aussi l'idée d'une vie propre de l'image : «J'espère avoir pu déjà participer sans le savoir à la réalisation d'une œuvre puissante et forte, qui vit d'elle-même, faisant écho à la solitude que j'ai laissée voir ». La puissance vitale de l'image va de pair avec l'impuissance à saisir autre chose qu'un fragment sans complétude possible. Le film s'ouvre d'ailleurs sous le signe de la Vénus à son miroir de Velázquez, qui met en avant une beauté en retrait, de dos, donc l'image réfléchie par le miroir que lui tend l'ange demeure indéfinie et vague, mystérieuse, absorbée dans ses propres pensées, alors même qu'elle semble interpeller le spectateur par son regard, au même titre que l'apparition du visage du modèle dans le plan suivant (fig. 9 et 10).

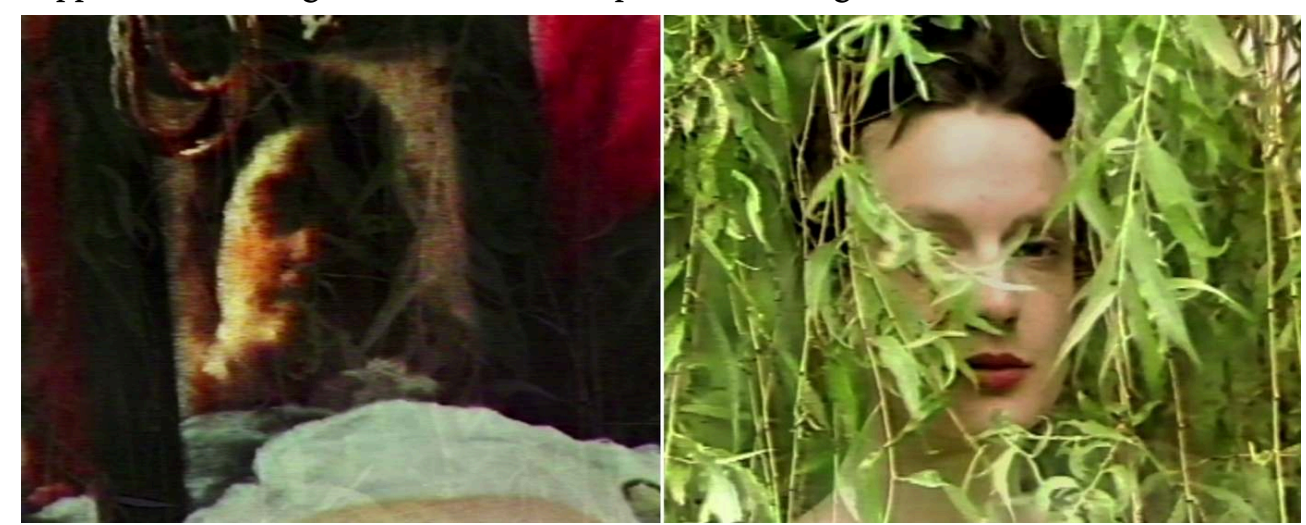

Fig. 9 et 10 

creusant entre autres l'idée d'une scission entre la parole et l'image. L'acte d'image schématique est défini par une forme de performativité, telle que la proposent par exemple les photographies de Cindy Sherman. Ces corps qui performent de manière assumée et remarquable une mise en scène de soi donnent une vitalité toute particulière au corps représenté. Dans le film de Roth, la caméra est l'outil qui permet de renforcer cette relation entre l'image et le corps, alors que la voix off vient renforcer sa vitalité et creuser le domaine de l'invisible. Car en effet, le corps en relief, pris dans un réseau de regards dont celui de la caméra, est également un corps-écran qui reflète les projections des autres, tout en réfléchissant pour son propre compte. L'acte d'image se dérobe, et c'est bien là que niche sa vitalité.

Quant à l'émouvant Ave Maria, ce n'est pas seulement son titre qui renvoie à la sacralité de l'acte d'image substitutif, mais le geste cinématographique qui lui est consubstantiel. En effet, telle une partition mystérieuse, tel un rituel dont on reconnaîtrait à peine les règles, le film rend interchangeables l'image et le corps, en élargissant celui-ci aux objets et à la nature : le seul corps présent dans le film est à son tour une image, celle d'une dame portant une couronne de fleurs et une faucille, sans doute une allégorie de l'été qui fait écho à la plénitude de la nature que nous montre le film. La puissance de cette interchangeabilité est homologue à la relation entre l'objet réel et son usage sacré que traduit le symbolisme religieux. Dans ce film-prière, l'objet est une référence à la parole sacrée et dans le même temps une figuration muette de celle-ci. À la fois cérébral et naïf, Ave Maria emporte par la simplicité de ces images fragiles, nues par rapport au contenu sacral qu'elles représentent. Symbolisant le transcendantal, les images incarnent le sacré et l'actualisent. Quelle meilleure « croyance » aux images que ce ciné-poème un peu mécanique, dont la simplicité touche à une paradoxale innocence? Croyance dans les puissances de l'image en mouvement mais aussi du montage, très pasolinien en ceci qu'il opère lui aussi des "actes d'images ", telles la multiplication des pains (fig. 11, 12, 13) ou la transition abrupte de l'arbre plein de fruits aux branches sèches (fig. 14, 15), qui peuvent rappeler le miracle du lépreux figuré dans L'Évangile selon Matthieu (1964) ${ }^{14}$ et la réinvention de la parabole du figuier stérile dans La Séquence de la fleur de papier (1967).

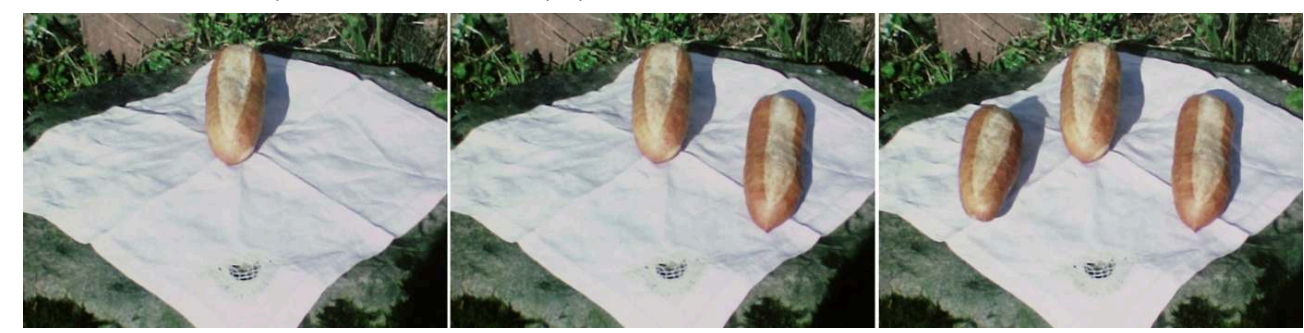

Fig. 11, 12 et 13 


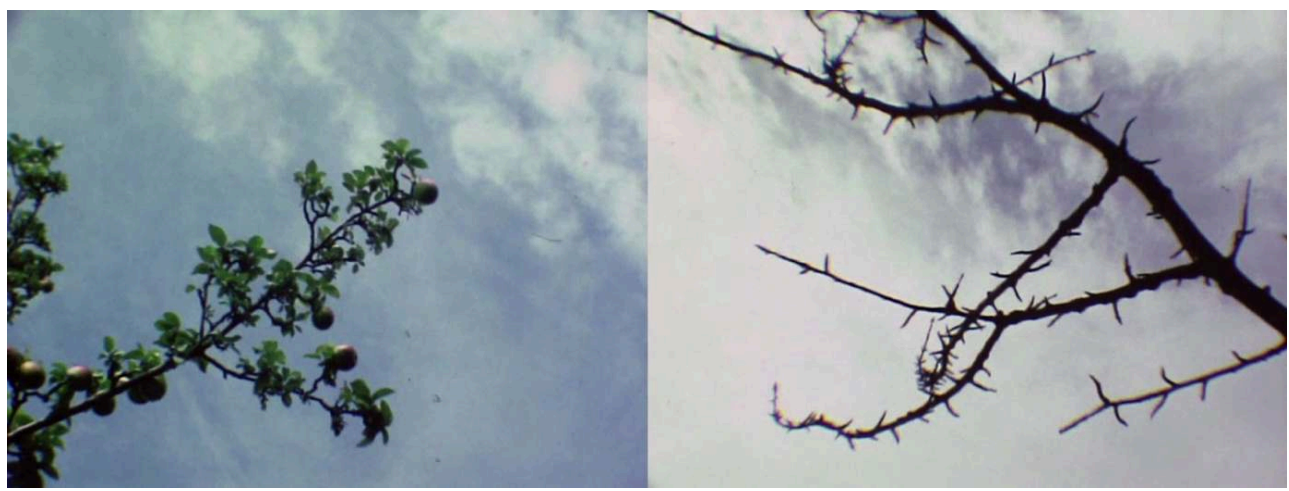

Fig. 14 et 15 qui fait appel aux puissances propres des images. Nous avons déjà eu l'occasion de constater les défis que Roth pose à l'image cinématographique, qui se traduisent également par un goût limité pour le formalisme. Cela n'est évidemment pas en contradiction avec l'idée d'une volonté d'expérimentation, qui se traduit par un certain minimalisme dans ses derniers films, jusqu'à l'objet trouvé à l'état " pur » : la bobine de found footage utilisée dans Le Pays fantôme (2019). L'acte d'image intrinsèque témoigne d'une action sur le regardeur, dont le paradigme fourni par Bredekamp est celui du mythe de Méduse déjà opératoire pour Siegfried Kracauer ${ }^{15}$ : les images de guerre, si elles peuvent pencher du côté de l'acte d'image substitutif quand elles sont utilisées pour la "guerre des images » (Bredekamp développe longuement ce point), relèvent de ce troisième type d'acte dans Les Yeux brûlés, car leur pouvoir de propagande est déjoué et redéfini par le regard porté sur ces images, que thématise et renforce le regard de Mireille. Ce que nous voyons, ce qui nous regarde: le titre de l'ouvrage de Georges DidiHuberman ${ }^{16}$ exprime bien cette ambivalence qui se retrouve dans l'acte d'image intrinsèque.

par Roth pros lealisés (es puissances du cinéma : deux films de montage, à savoir les deux fragments avec Raoul Coutard et Pierre Schoendoerffer (2017) issus des rushes des Yeux brûlés; deux captations transfigurées, films d'images théâtrales mélangées à d'autres grâce à des surimpressions: Écoute Israël et La Joie. Je m'arrêterai brièvement sur les deux derniers, dont le minimalisme des moyens d'expression permet de mettre à nu des partis pris fondamentaux, en insistant notamment sur l'acte de création, sur le geste du cinéaste qui choisit d'inscrire des surgissements d'images et de sons rarement illustratifs par rapport au texte lu, plus souvent évocateurs, qui résonnent avec la voix des acteurs-lecteurs et ouvrent d'autres niveaux discursifs. Les actes que présente Écoute, Israël sont dérivés d'autres matériaux: aussi bien la lecture que les images d'archives mettent en scène des éléments personnels de l'univers de l'auteur. L'image en surimpression est elle-même à la fois archive et rêverie ${ }^{17}$, réalité brutale et imagination condensant une multitude de fragments. L'éclatement et la déliaison des images par rapport aux sons se retrouve tout particulièrement dans le texte discontinu et fragmentaire écrit par Roth, d'autant plus remarquable qu'il paraît fluide à la syntaxe comme à la lecture. Dès lors, ce flux discontinu d'images et de sons transporte le spectateur dans un territoire incertain qui contredit de manière perturbante la simplicité de la mise en scène et des procédés filmiques. "Actes d'image intrinsèques ", 
les images-éclats soulignent à la fois la puissance d'apparition et l'incomplétude propres à l'image. L'éblouissement établit donc le moment incommensurable - car entièrement qualitatif - où l'acte d'image atteint son paroxysme, déstabilise son spectateur, affirme l'insoutenable en s'exposant à sa négation. Tout comme la défiguration et la déformation ont permis à Lyotard ${ }^{18}$ de poser les bases d'une pensée esthétique du "figural ", de la limite et de la transgression propre à l'acte d'image, Roth souligne par ses derniers films que la puissance figurale de l'image réside paradoxalement dans les formes de sa négation.

La première partie de l'œuvre du cinéaste analysée ici - dans la mesure très relative où il fait sens d'établir ces différences en postulant des "étapes » là où il n'y a le plus souvent que des versants d'une personnalité à la rencontre des conjonctures historiques - semble donner une réponse quelque peu différente, plus sensible à une quête de beauté des images; nous nous y arrêtons ici pour montrer la diversité des formes que prend l'acte d'image intrinsèque. En effet, il y a dans Henri Alekan, des Lumières et des Hommes (1986), un paradoxe que seul ce troisième acte d'image permet d'expliquer, en déployant les puissances propres de la mise en scène cinématographique. Dès le début du film, Alekan revendique l'importance de l'imagination, la capacité de créer un univers cinématographique autonome, par une sorte d'architecture de la lumière. Mais l'image de l'entretien de Laurent Roth, en noir et blanc, porte une idée de la lumière (et de la beauté) tout autre: Roth tourne à l'extérieur, en déléguant une part importante de la maîtrise du cinéaste au hasard, en laissant se rencontrer la lumière naturelle et une pellicule périmée, en insistant sur l'image comme surgissement. L'événement de la lumière n'est pas l'émanation du sujet créateur mais le produit d'une rencontre avec le monde. D'ailleurs, à la différence d'Alekan, Laurent Roth se définit " plus archéologue qu'architecte ${ }^{19}$ ".

S'il construit une forme, Roth garde le lien avec la matérialité de l'image, en accueillant l'imprévu comme les changements de lumière à l'extérieur. L'intrusion du luxmètre dans la scène (fig. 16) souligne cette variabilité de la lumière qui transparaît de manière frémissante grâce au cadre naturel choisi par Roth et par les taches de lumière qui transpercent le feuillage. L'image en mouvement est ici le lieu d'une modulation qui révèle sa nature d'image changeante, littéralement mouvante. Le film de Roth est un hommage d'autant plus vibrant qu'il est personnel et lié à sa sensibilité de cinéaste. Soucieux à la fois de construire un dispositif filmique et de l'ouvrir à l'écart et à l'inachevé, Roth rejoint par d'autres chemins la nécessité d'invention et d'expérimentation que revendique le célèbre directeur de la photographie. 


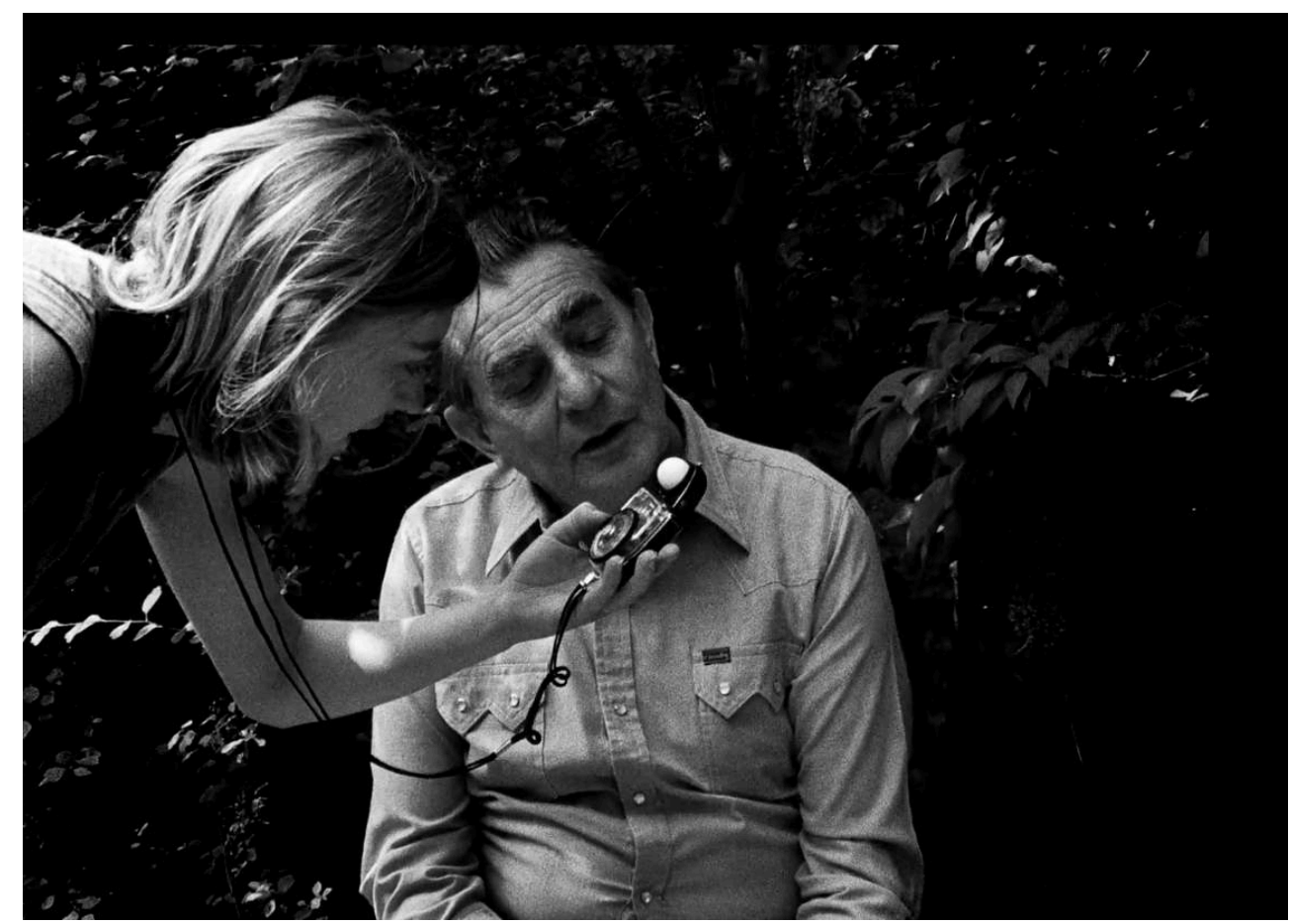

Fig. 16

\section{L'image et la parole}

Nous avons étudié la hantise de l'éblouissement qui constitue une sorte d'image matricielle et d'image-limite de l'univers de Laurent Roth ; nous avons ensuite creusé le versant "agissant" de l'image à l'aide des réflexions de Horst Bredekamp. Outre l'impuissance de l'image, sa puissance destructrice ou autodestructrice, sa performativité, il nous reste à creuser un dernier territoire, que nous avons évoqué à plusieurs reprises, à savoir les relations qu'entretiennent l'image et la parole dans le cinéma de Roth. Nous ne pourrons ici qu'avancer quelques hypothèses, en gardant tout particulièrement à l'esprit les premiers films du cinéaste. En effet, si l'on repense au propos du cinéaste sur Les Yeux brûlés, insistant sur les limites de l'image, on pourrait en tirer - en partie à raison - l'idée que Laurent Roth lui préfère la parole, en reproduisant ainsi une hiérarchie souvent réactivée par la tradition hébraïque. Ce serait pourtant méconnaître l'attention pour l'image qui est la sienne, $y$ compris là où il en fait un usage très parcimonieux mais d'autant plus percutant, par exemple dans $L a$ Joie, comme nous l'avons vu. Le flux de parole se déploie alors dans toute sa puissance, et l'image vient l'intercepter par moments, par éclats (La Joie) ou étirements (Raoul Coutard, J'ai pas une tête de mort et Pierre Schoendoerffer, La Peine des hommes), par fulgurances donc. L'image n'est pas absentée mais rendue à son pouvoir de surgissement, et le rapport à la figuration n'est pas résolu par le refus ou par l'iconolâtrie, mais creusé dans sa complexité anthropologique et esthétique.

21 La parole joue un rôle autrement fondamental, par sa présence comme par son absence. Il suffit de songer à Ave Maria, dont la version originale était accompagnée « live » d'un chant composé par Giacinto $\mathrm{Scelsi}^{20}$ : la parole se fait image, mais aucune représentation directe du corps humain ou divin n'est donnée. Le chant ajouté lors de la remasterisation du film en 2018, suggère à son tour une forme de prière que les 
images seules proposaient à leur manière ${ }^{21}$. Homologues comme dans la première version, déliés dans la deuxième, les parcours de la bande son et de la bande image se déploient en parallèle, tel un duo poétique ou spirituel, construisant une harmonie assez unique dans l'œuvre de Roth. La parole ne vient pas résoudre ce que l'image laisse inaccompli, car elle aussi est hantée par une forme d'absence, par le silence des choses, des êtres et des images. Toute image n'est pas image de Dieu, et la parole des hommes n'est pas le Verbe: la matière filmique chez Roth n'en demeure pas moins une confrontation à la transcendance, qui trouve dans la fragmentation et l'incomplétude son horizon matériel, dans l'articulation et le flux son horizon spirituel.

La solidarité entre les surgissements respectifs de l'image et de la parole se manifeste de manière plus programmatique dans Ranger les photos. Dominique Cabrera y énonce l'ambivalence du geste photographique, en insistant sur la douceur et la violence, sur la légèreté et la gravité du geste photographique, à la fois simple, instantané mais définitif, consigné à l'Histoire, ou du moins à l'histoire personnelle. Cette ambivalence trouve sa forme dans le dispositif de réalisation: tourné-monté, coréalisé, le film prévoit une série de douze plans fixes qui débutent et s'achèvent par un fondu. L'image et le son émergent ensemble du noir et du silence, et cette unité est renforcée par le statut fortement descriptif de la bande son, qu'annonce le plan d'ouverture du film où les mains de Cabrera composent avec des lettres de Scrabble le titre du film, tandis que sa voix l'énonce. En effet, Ranger les photos va dépeindre pour l'essentiel l'action qu'indique son titre performatif ; mais ranger, c'est plutôt toucher et évoquer, par la parole, des récits qui viennent encadrer ou déborder les images. Ces récits, ce sont des bribes autobiographiques, accompagnées d'une interrogation sur la photographie.

Fragmentaire, le film dessine une unité sentimentale, une atmosphère cohérente, basées sur une certaine manière de raconter qui intègre les hésitations et la gestuelle, un rapport physique aux photos qui permet d'insuffler la parole et d'opérer la connexion au passé et à la transmission. La tautologie du plan initial, où l'on entend Dominique dire, ou lire les lettres de Scrabble qu'elle cherche et dispose, jusqu'à énoncer le titre, cette tautologie n'est qu'apparente, car la durée du plan permet de matérialiser le titre sous nos yeux, par un processus manuel, en insistant sur l'action, la quête, la gestuelle. "Je ne trouve plus le $t$ » (à moins que ce soit «le thé », vu qu'elle joue avec les mots...), dit-elle vers la fin du plan, quand il n'y a plus rien à découvrir de cet intitulé que l'on devine clairement (fig. 17) ; puis, une fois trouvée la dernière lettre, Dominique lit le titre qu'elle a composé : la parole est donc prise dans un double jeu d'écriture et de lecture, à la fois actif et passif, d'immédiateté et d'élaboration. 


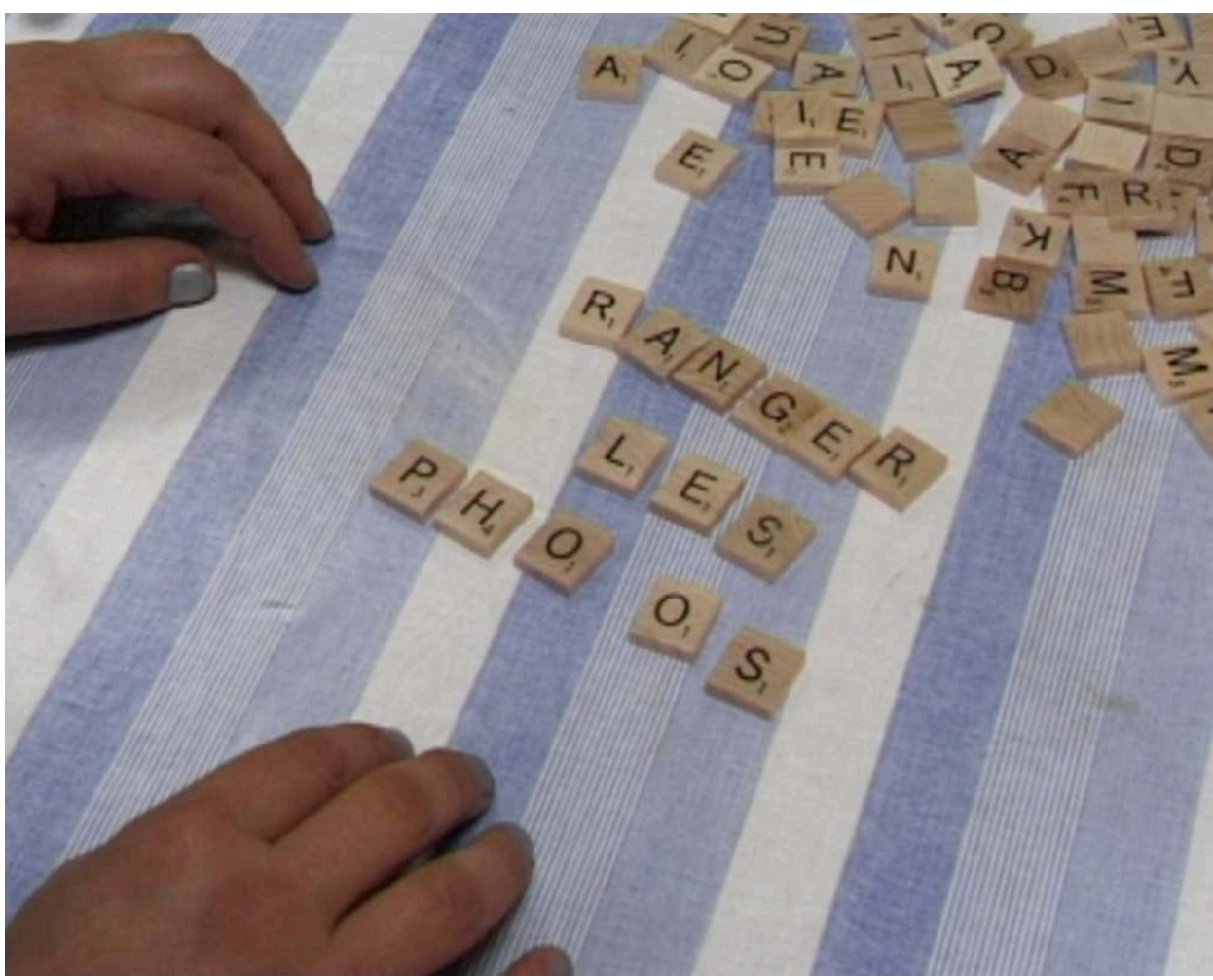

Fig. 17

Le résultat, c'est le processus - voilà ce que semble nous annoncer le plan liminaire, à la fois portion de générique et partie intégrante du film. « Enlever et mettre le vernis à ongles » : ce geste parallèle au travail sur les photos, qui donne le thème du onzième « tableau » (j'emploie ce terme dans la mesure où la structure du film peut faire penser à une réinvention des douze «tableaux » de Vivre sa vie de Jean-Luc Godard, 1962), est réflexif en ce qu'il évoque le principe de la mise en scène, la vanitas du travail répétitif de la mécanique photo/cinématographique, le recouvrement qui est propre au geste de la reproduction mécanique. Tel un moulage, l'étalement du vernis cache, rend visible et fictionnalise. "Il ne reste plus qu'à mettre un peu de vernis maintenant ", dit-elle en riant en off (fig. 18), juste avant le portait final, grave cette fois-ci, et immobile (fig. 19), point d'orgue d'un film construit une fois de plus en fonction d'un paroxysme final, d'une forme d'éblouissement subtile et apaisée ${ }^{22}$. Le corps et la voix, le fragment et le portrait se donnent le relai pour interroger un rapport à la représentation toujours fragile, toujours indirect car filtré par l'esprit et par le dispositif cinématographique.

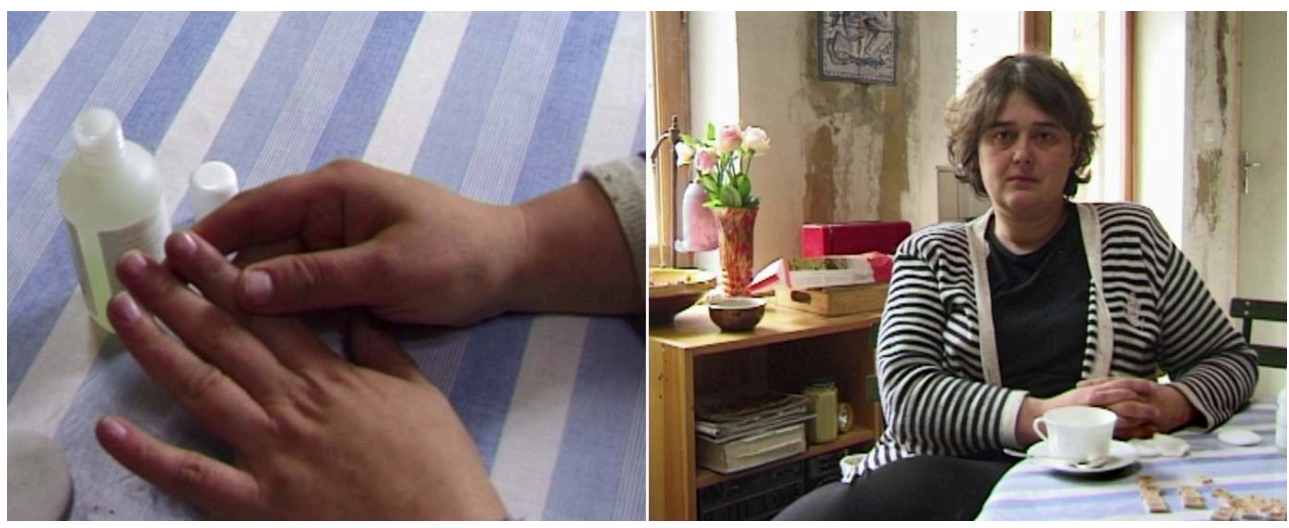

Fig. 18 et 19 

(transcendance, affaiblissement, dépossession), à l'éblouissement comme hantise fondamentale, à la fragilité constitutive du geste artistique qui rend possible l'acte esthétique. On comprend mieux la nécessité de changer de mode opératoire dans chaque film, tout en gardant le cap d'une mise en tension des éléments en jeu, dans la mise en scène, entre les images et entre les sons : noir et blanc, couleur; plans longs ou plans-séquences, surimpressions, faux raccords ; écarts entre image et son. Réussir cet entrechoc signifie construire une structure au bord de l'abîme, fêler la continuité de la chaîne filmique pour encourager un rôle autrement actif du spectateur, l'inviter à prendre part à une forme ouverte, dialogique, essayiste - la notion de « film-essai » est tout à fait productive, de ce point de vue, pour comprendre l'œuvre de Roth. Celle-ci ne s'installe pas dans l'univers de l'éblouissement, comme une partie du cinéma expérimental, mais elle le signifie comme étant sa dimension originelle, matricielle. L'exigence face à l'image est d'autant plus forte qu'elle affiche souvent la plus grande simplicité, y compris quand la composition du cadre est recherchée comme dans ses premiers films. Cette simplicité, est celle de l'évidence muette de toute image, de l'acceptation de l'insuffisance de toute parole humaine, de l'intégration du hors champ dans le processus de création.

Roth se trouve en tant que cinéaste lorsqu'il met sa lucidité, son intelligence et ses ruses de metteur en scène au service du nœud esthétique qui le hante, tel un principe d'incertitude qui met au défi l'unité de la composition. Un film «barbare » (il le définit lui-même de la sorte) comme Marie ou le retour représente bien ce principe et cet acheminement, par juxtaposition de fragments, qui met en tension la construction d'une structure forte (en cinq parties) et le point-limite qui la met en branle. Le retour $\mathrm{du}$ père coïncide avec la perte de l'innocence paradisiaque figurée par exemple par l'apparition, au milieu du film et entre deux fondus au blanc, de l'éblouissant Paradis terrestre (vers 1607-1608) de Brueghel l'Ancien. Comme le tableau, le film pose une réflexion sur le bien et le mal qui reste en filigrane dans toute l'œuvre du réalisateur, et que confirme l'emploi de morceaux tirés de Phèdre de Racine dans le court-métrage. On comprend mieux la brutalité de la fin de Marie ou le retour, qui brise la série raffinée de références culturelles que propose le reste du film par l'irruption brutale de la guerre. L'éblouissement face à l'Histoire correspond, au milieu du film, à celui que provoque l'Art, et avec lequel il partage une complicité souterraine, une force de dépossession inquiétante. Au cœur de la quête esthétique ininterrompue de Laurent Roth, comme le démontreront plus tard ses films plus directement autobiographiques, niche alors une quête existentielle, aux accents métaphysiques.

\section{NOTES}

1. Laurent Roth, «Mère, je brûle! L'éthique du documentaire », Cahiers du cinéma, $\mathrm{n}^{\circ} 489$, mars 1995. 
2. Robert Bresson, Notes sur le cinématographe, Paris, Gallimard, 1975, p. 25. Cité par Laurent Roth dans Michèle Levieux, «Une jeune fille et la mort », entretien avec Laurent Roth, L'Humanité, 5 avril 2019, en ligne : https://www.humanite.fr/une-jeune-fille-et-la-mort-670464.

3. [NDL] Voir dans ce volume: Roger-Philippe Della Noce-Bertozzi, «Laurent Roth, poète de l'immanence de la transcendance ».

4. Dario Marchiori, « Brevitas moderniste. Bref excursus sur la temporalité contrariée du cinéma expérimental », Les Formes brèves, actes des Journées d'études de l'ASSIC, Université Paris III Sorbonne Nouvelle, 2007.

5. "Étymol. et Hist. 1. Mil. $\mathrm{xv}^{\mathrm{e}}$ s. esblouissement "état de l'esprit en proie à une vive admiration" » (G. Chastellain, Exposition sur vérité mal prise, dans Euvres, éd. Kervyn de Lettenhove, t. 6, p. 262.) ;

2. 1539 «trouble de la vue provoqué par une cause interne, souvent accompagné de vertiges » (Est.) ; 3. av. 1549 «trouble momentané de la vue causé par une lumière trop vive » (Marguerite de Navarre, La Nativité de Jésus-Christ, 600 dans Comédies, éd. F. E. Schneegans). Dér. du rad. du part. prés. de éblouir*; suff. -ment* " (https://www.cnrtl.fr/etymologie/\%C3\%A9blouissement, consulté le 30 juin 2020). Il est intéressant de remarquer l'origine militaire du terme, particulièrement appropriée pour réfléchir aux Yeux brûlés mais plus largement à l'œuvre de Roth.

6. Serge Daney, "Les Yeux brûlés, ou la métaphysique guerrière ", dans Libération, 10 octobre 1986 ; repris dans La Maison cinéma et le monde 3. Les Années Libé 1986-1991, Paris, P.O.L, p. 70-71.

7. Régis Durand, «La lumière même», dans Régis Durand et Dominique Baqué (dir.), Éblouissement, catalogue d'exposition, Paris, Jeu de Paume, 2004. Dans son article, Régis Durand s'appuie également sur l'article de Jonathan Crary, "Aveuglante lumière », dans Aux origines de l'abstraction 1800-1914, catalogue d'exposition (Musée d'Orsay 2003-2004), Paris, Réunion des Musées Nationaux, 2003.

8. Ibid.

9. Laurent Roth, «Entretien avec Laurent Roth », fait à Paris le $1^{\mathrm{er}}$ septembre 1986. [NDL] Repris dans ce volume sous le titre : «Auto-entretien. Paris, 1er septembre 1986 ».

10. L'historien de l'art Horst Bredekamp va jusqu'à affirmer que l'iconoclaste «est plus fortement gouverné par les images que l'adorateur de celles-ci » (H. Bredekamp, Théorie de l'acte d'image [2010], Paris, La Découverte, 2015, p. 194).

11. Cf. "La Vague et le langage », dans « Laurent Roth. Courts-métrages : L'intégrale » (2019, La Huit). [NDL] : cet entretien a été repris et augmenté en vue de la présente publication sous le titre «Commencer en cinéma ».

12. Horst Bredekamp, Théorie de l'acte d'image, op. cit.

13. John L. Austin, Quand dire, c'est faire [1962], Paris, Éditions du Seuil, 1970.

14. Lors de la Journée d'études dédiée à Laurent Roth (CinéAquarium, Lyon, le 14 novembre 2019), en sa présence, le cinéaste a rappelé que le générique noir sur fond blanc des Yeux brûlés était inspiré du film de Pasolini.

15. Siegfried Kracauer, Théorie du film: la rédemption de la réalité matérielle [1960], Paris, Flammarion, 2010 ; cf. notamment «La tête de Méduse », p. 429-431 ("L'écran de cinéma est le bouclier poli d'Athéna ", p. 430).

16. Georges Didi-Huberman, Ce que nous voyons, ce qui nous regarde, Paris, Éditions de Minuit, 1992.

17. Sur ce lien entre surimpression et rêverie, voir Marc Vernet, Figures de l'absence, Paris, Cahiers du cinéma, 1988.

18. Jean-François Lyotard, Discours, Figure, Paris, Klincksieck, 1971.

19. Michèle Levieux, « Une jeune fille et la mort », entretien avec Laurent Roth, op. cit.

20. Voir, ou plutôt écouter : Giacinto Scelsi, Musique sacrée, 33T, FY, 1985 (pièce originale : Three Latin Prayers : Ave Maria, Pater Noster, Alleluja, 1970). 
21. Antonio Ruiz-Pipo, Canço d'etsequeiar, dans Cinco tonadas, album réalisé à partir de «textes populaires de différentes régions d'Espagne» (1971): https://data.bnf.fr/fr/14567064/ antonio_ruiz-pipo_cinco_tonadas/.

22. Comme l'exprime le réalisateur : «J'ai construit tout le film mentalement au tournage pour aboutir à ce plan que j'avais prévu et désiré » (mail à l'auteur, le 23/11/2020). La puissance de ce plan final, sa gravité qui détonne, se devaient sans doute d'être préparées et enrichies grâce à la légèreté sérieuse des onze tableaux précédents: rare capacité de réinvention d'une tradition noble comme celle du portrait, à la fois démocratisée et conservée dans toute sa profondeur réaliste, psychologique et métaphysique.

\section{RÉSUMÉS}

À travers l'analyse des premiers films de Laurent Roth, l'article avance l'idée que l'éblouissement est un sujet fondateur qui se retrouve dans ses premiers films et qui établit un rapport à l'image basé sur le doute, la réflexivité, voire la défiance. En s'appuyant sur «la théorie de l'acte d'image » avancée par Horst Bredekamp, l'article souhaite ensuite aborder la puissance agissante de l'image chez Laurent Roth, en pointant différents versants de ses "actes d'images». Troisièmement, l'article interroge l'éventail des relations entre l'image et la parole, l'une interrogeant l'autre, révélant ses fragilités et ses puissances. La violence de l'émerveillement allant de pair avec la force de reconnaître l'impuissance de l'art.

Through the analysis of Laurent Roth's early films, the article puts forward the idea that glare is a founding subject that is found in his first films and that establishes a relationship to the image based on doubt, reflexivity, even defiance. Based on Horst Bredekamp's Theory of the Image Act, the article then addresses the active power of the image in Laurent Roth's work, pointing out different aspects of his "acts of the image". Thirdly, the article questions the range of relations between image and word, one questioning the other, revealing its fragilities and powers. The violence of wonder goes hand in hand with the strength to recognise the powerlessness of art.

\section{AUTEUR}

\section{DARIO MARCHIORI}

Dario Marchiori, maitre de conférences en Histoire des formes filmiques à l'Université Lyon 2, travaille sur le cinéma moderne et sur les relations entre documentaire, film-essai et cinéma expérimental. Il a édité un recueil d'écrits sur le cinéma d'Alexander Kluge, L'Utopie des sentiments (PuL, 2014) et codirigé Expanded Cinema (Garnier, 2015) et Lisières esthétiques et culturelles au cinéma (Garnier, 2020). 